\title{
ORAL HYGIENE PRACTICES OF FIRST AND FINAL YEAR DENTAL STUDENTS AT FOUNDATION UNIVERSITY: A COMPARATIVE STUDY
}

\author{
Emaan Ahsin, Sadia Ahsin \\ Foundation University College of Dentistry, Islamabad Pakistan
}

\begin{abstract}
Objective: To compare personal oral hygiene practices between first and final year dental students of Foundation University College of Dentistry (FUCD).

Study Design: Cross sectional study.

Place and Duration of Study: Foundation University College of Dentistry, Islamabad, from Mar to May 2019.

Methodology: A pre-structured questionnaire was distributed to students of first and final year BDS classes with questions related to seven basic oral hygiene practices. These included frequency of teeth cleaning, time spent in cleaning teeth, tools used for cleaning teeth, frequency of changing toothbrushes, use of secondary plaque control methods, whether or not students cleaned their tongue, and how often they visited a dentist.

Results: Only 2 out of 7 practices, (i.e. more frequency of brushing, 45/52 (86.5\% in final year vs 25/47 (53.1\% in first year and usage of floss 22/52 (42.3\%) in final year vs 9/47 (19.1\%) in first year) were found to be significantly better in final year students when compared to first year BDS students with a $p$-value of 0.012 and 0.04 respectively. The remaining differences did not reach statistical significance.

Conclusion: When compared with first year BDS students, only 2 out of 7recommended oral hygiene practices were found to be better in final year students. Therefore, there is a need for greater emphasis on oral hygiene practices at undergraduate educational years.
\end{abstract}

Keywords: Brushing, Compare, Dental students, Flossing, Oral hygiene practices.

This is an Open Access article distributed under the terms of the Creative Commons Attribution License (http://creativecommons.org/licenses/by/4.0), which permits unrestricted use, distribution, and reproduction in any medium, provided the original work is properly cited.

\section{INTRODUCTION}

The American Dental Association (ADA) defines oral health as "a functional, structural, aesthetic, physiologic, and psychosocial state of well-being and is essential to an individual's general health and quality of life"1. According to the WHO, despite the global improvements, oral diseases still present a major problem for developing countries ${ }^{2}$. To this, Pakistan is no exception. Along with oral health issues, Pakistan also faces multiple socioeconomic challenges. Hence, to preserve resources, it is vital to lessen the burden posed by oral diseases ${ }^{3}$. This starts with oral hygiene.

Oral hygiene is the state of keeping one's mouth, teeth, and gums clean and free of disease. Various methods and practices exist to achieve good oral hygiene. Key practices include brushing teeth at least twice a day, cleaning between

Correspondence: Emaan Ahsin, Foundation University College of Dentistry, Islamabad Pakistan

Received: 15 May 2020; revised received: 28 Jul 2020; accepted: 12 Aug 2020 teeth, visiting a dentist regularly, using mouth wash, flossing, etc.

Dental students play a vital role in promoting and creating awareness about correct oral hygiene practices, it should therefore be logical that dental students have exceptional oral hygiene practices. However, studies show that this is not always the case; a study done in Yemen ${ }^{4}$ showed the markedly poor oral hygiene practices of dental students. Another study done in Sudan also showed similar results 5 . Regardless of the subpar oral hygiene practices of dental students, it may not be fair to assume that these practices do not differ amongst senior and junior undergraduate classes of dentistry. The few studies that compare preclinical and clinical dental students' self-perceived oral hygiene practices have inconsistent results, for example, a study in India showed that there were limited statistically significant differences in oral hygiene practices of students in pre-clinical and clinical years ${ }^{6}$, while a study done in Istanbul proved that students in 
clinical years indeed had better practices and knowledge?

With this background, this study was planned to record and compare the oral hygiene practices of first and final year classes of dentistry at Foundation University College of Dentistry. This study hypothesized that final year students have better oral hygiene practices than first year students as they have greater knowledge of dental practices, oral hygiene, and more practical experience as they interact with patients.

\section{METHODOLOGY}

This cross-sectional study was carried out at Foundation University College of Dentistry (FUCD) from March to May 2019. Approval from the ethics review committee of the University (ERC approval letter No. FF/FUMC/215-Phy/19) was obtained prior to conducting the study. The inclusion criteria were students from first and final year Bachelor of Dental Sciences (BDS) students while those who refused to informed consent were excluded.

The total strength of each class at FUCD is maximum 50-52 students. Since it was a pilot study being done at FUCD only, therefore all students from first year BDS (total 50) and final year BDS (total 52) were included in the study. The sampling method used was non-probability convenience sampling. After literature review, a modified and self-administered questionnaire was adapted from Philipp et al and Inaam et al 8,9 . It contained seven items carrying questions related to the main practices of oral hygiene. These were frequency of teeth cleaning, time spent cleaning teeth, tools used for cleaning teeth, frequency of changing toothbrushes, use of secondary plaque control methods, whether or not students cleaned their tongue, and how often they visited a dentist. The participants were kept anonymous. They were asked to honestly select one answer out of a range of options given.

The data from all distributed questionnaires was entered and analyzed through SPSS-24. The frequencies and percentages of responses for each question for all participants were calculated.
Chi square test was used to determine significant difference at a $p$-value of $<0.05$.

\section{RESULTS}

Of 102 participants, with age range 18-25 years, 99 completed the questionnaires, 47 participants were from first year and 52 were from final year with total 9 males and 90 females. Of the 7 areas of oral hygiene practices highlighted in the questionnaires, i.e. teeth cleaning, time spent cleaning teeth, tools used for cleaning teeth, frequency of changing tooth brushes, use of secondary plaque control methods, whether or not students cleaned their tongue, and how often they visited a dentist, only 2 were found to significantly better in final year BDS students using ChiSquare test (table). This included frequency of teeth cleaning twice a day and use of secondary plaque control methods, specifically flossing. For teeth cleaning it was found that $45 / 52(86.5 \%)$ of final year BDS students brushed twice a day, while in first year 25/47 (53\%) brushed twice

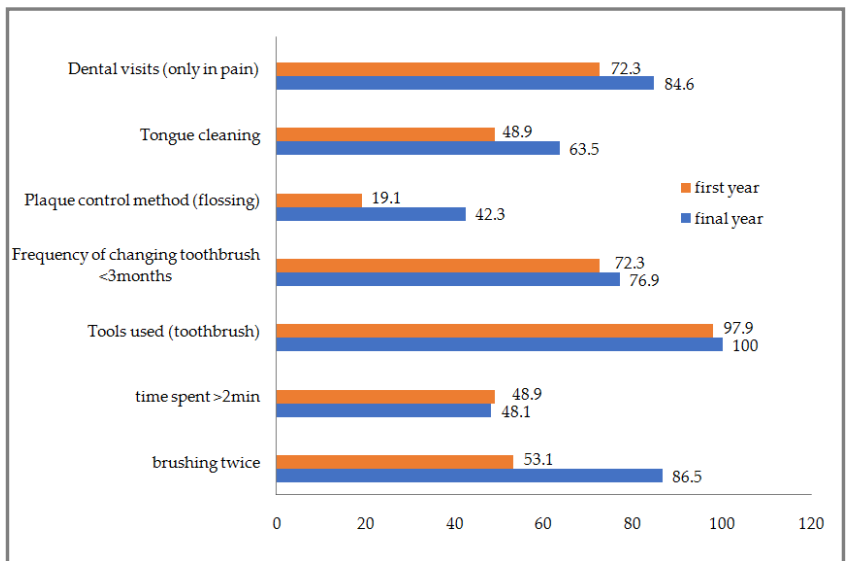

Figure: Comparison of percentage responses of first and final year BDS students in 7 areas of oral hygiene practices.

a day. The difference was found to be significant reaching a $p$-value of 0.01 . For flossing the difference in the two classes of students was found to have a $p$-value of 0.041 , wherein final year it was found that $22 / 52(42.3 \%)$ of students floss regularly while in first year only $9 / 47(19 \%)$ of students used dental floss. The remaining five areas also apparently showed better results in final year BDS students but these did not reach statistical significance (figure). 


\section{DISCUSSION}

Professional Dental colleges play a vital role in shaping a student's perception on oral health and hygiene, and hence this effects how they influence their patients as oral health care provi- oral hygiene practices in 5 out of 7 parameters estimated in the questionnaire. Literature shows 7,10 , that with increasing levels of dental education, there is a significant difference in oral hygiene, with clinical students having better practices.

Table: Results showing difference of oral hygiene practices in first and final year BDS students using Chi square test.

\begin{tabular}{|c|c|c|c|}
\hline & Final Year $(n=52)$ & First Year $(n=47)$ & $p$-value \\
\hline \multicolumn{4}{|c|}{ Frequency of teeth cleaning } \\
\hline Once a day & $5(9.6 \%)$ & $20(42.5 \%)$ & \multirow{3}{*}{ Approx. 0.012} \\
\hline Twice a day & $45(86.5 \%)$ & $25(53.1 \%)$ & \\
\hline More than twice a day & $2(3.8 \%)$ & $2(4.2 \%)$ & \\
\hline \multicolumn{4}{|c|}{ Time spent cleaning teeth } \\
\hline Less than 2 minutes & $16(30.8 \%)$ & $20(42.5 \%)$ & \multirow{3}{*}{ Approx. 0.213} \\
\hline 2 minutes & $25(48.1 \%)$ & $23(48.9 \%)$ & \\
\hline More than 2 minutes & $11(21.1 \%)$ & $4(8.5 \%)$ & \\
\hline \multicolumn{4}{|l|}{ Tools for cleaning teeth } \\
\hline Tooth brush & $52(100 \%)$ & $46(97.9 \%)$ & \multirow{3}{*}{ Approx. 0.732} \\
\hline Fingers & $1(1.9 \%)$ & $1(2.1 \%)$ & \\
\hline Miswak & $4(7.7 \%)$ & $1(2.1 \%)$ & \\
\hline \multicolumn{4}{|c|}{ How often do you change your tooth brush? } \\
\hline Every 3 months & $40(76.9 \%$ & $34(72.3 \%)$ & \multirow{2}{*}{ Approx. 0.304} \\
\hline Every 6 months & $12(23.1 \%)$ & $13(27.6 \%)$ & \\
\hline \multicolumn{4}{|c|}{ Which secondary methods for plaque control do you use? } \\
\hline Dental floss & $22(42.3 \%)$ & $9(19.1 \%)$ & \multirow{5}{*}{ Approx. 0.041} \\
\hline Interdental brushes & - & $3(6.4 \%)$ & \\
\hline Mouth wash & $37(71.1 \%)$ & $22(46.8 \%)$ & \\
\hline Tooth picks & $5(9.6 \%)$ & $6(12.8 \%)$ & \\
\hline None & $1(1.9 \%)$ & $11(23.4 \%)$ & \\
\hline \multicolumn{4}{|c|}{ Do you clean your tongue? } \\
\hline Yes & $33(63.5 \%)$ & $23(48.9 \%)$ & \multirow{3}{*}{ Approx. 0.634} \\
\hline No & $5(9.6 \%)$ & $8(17 \%)$ & \\
\hline Sometimes & $14(26.9 \%)$ & $16(34 \%)$ & \\
\hline \multicolumn{4}{|c|}{ How often do you visit a dentist? } \\
\hline Only in problem & $44(84.6 \%)$ & $34(72.3 \%)$ & \multirow{3}{*}{ Approx. 0.614} \\
\hline Once in three months & $1(1.9 \%)$ & $4(8.5 \%)$ & \\
\hline Once in six months & $7(13.5 \%)$ & $9(19.1 \%)$ & \\
\hline
\end{tabular}

ders 5 . It has been shown that the behavior of dentists and their attitudes towards their own oral health shows their understanding of the importance of preventive dentistry and good oral health and hygiene ${ }^{7}$. While there have been some studies done on the oral hygiene practices in Pakistan, there have not been many studies done evaluating the personal oral hygiene practices of dental students, specifically.

Present study showed no significant difference between first and final year dental students
This could be due to the fact that students in clinical years not only have more knowledge about oral hygiene, but also have practical experience and hence are aware of the importance of oral hygiene. It may be interesting to note that similar studies done in western countries specifically ${ }^{7}$ showed significant differences in oral hygiene practicesand also better oral hygiene overall. However, there have been studies done in India6, Yemen ${ }^{11}$, and Sudan ${ }^{5}$ which contradict this result. Indeed, a study done by Swathi et al in $2015^{12}$ 
showed that preclinical students had better oral hygiene practices.

In present study, it was found that there was a significant increase in frequency of teeth cleaning as $86.5 \%$ of final year students revealed that they brushed twice a day as compared to only $53.1 \%$ in first year students. This is in accordance with a study done in Jordan ${ }^{13}$. This however contradicts a study done by Gupta et al ${ }^{6}$ which showed no significant difference between brushing frequency of preclinical and clinical students. Likewise, it also contradicts a study done in India12, which showed opposite results; with $45.1 \%$ and $39.7 \%$ of preclinical and clinical year students brushing twice a day respectively.

The second area which showed significantly better practices amongst final year students in comparison to first year was use of secondary plaque control methods, specifically, flossing. In first year, only $19.1 \%$ of the students used dental floss, which was similar to non-dental university students of Italy where authors found that only $15 \%$ of students used dental floss ${ }^{14}$. The reason for this low percentage in current study could be the lack of sufficient knowledge which is comparable to that of university students of any other major. In the current study $42.3 \%$ of final year students used dental floss, which was similar to the results found in India (46.1\%) ${ }^{15}$ and Ankara $(37.3 \%)^{16}$. This difference is in accordance to few other studies ${ }^{17,18}$. However, Barrieshi et al ${ }^{19}$ found no significant difference in flossing despite having an almost quadruple difference in brushing frequency between preclinical and clinical years of dental school. While it is a positive sign that the use of floss increased over the years of education in the current study, it was still less than the results found in dental students of other countries ${ }^{17}$. The minimal utilization of flossing can be attributed to the general lack of knowledge and awareness about flossing.

The present study found no significant difference in time spent cleaning teeth for first and final year students; approximately $48 \%$ of both classes spent 2 minutes brushing. This is similar to the findings of a study done in Pakistan ${ }^{9}$. There was also no significant difference found in frequency of dental visits. A majority in both classes stated that they only visited a dentist if needed (84\% for final year and $72 \%$ for first year). Similar results were found in a study done in India 6 , and the opposite findings from a study done in Jordan ${ }^{13}$. Studies done on assessing the oral hygiene of the general population of Pakistan also showed that people generally did not feel the need to visit a dentist regularly ${ }^{20}$ reason could be that people, including dental students, do not understand the importance of frequent checkups. For "tools used for cleaning teeth" close to $100 \%$ of students from both classes used a toothbrush. $72 \%$ of first year students and $76 \%$ of final year students were found to replace their toothbrush every 3 months, sim-ilar to the findings of a study done in Jaipur ${ }^{6}$. While $14.6 \%$ more students cleaned their tongues in final year compared to first year, the value though appreciable, was not found to be statistically significant. While this finding is similar to a study done by Gupta et $a l^{6}$ and Inaam et $a l^{9}$, it is important to note that percentages of cleaning tongue were found in those studies much higher (close to $80 \%$ ) as compared to present study (63.5\% in final year).

Overall, present study found no significant improvement in personal oral hygiene practices between first and final year dental students. This could be attributed to the lack of importance given to oral hygiene nationwide. A study in Mianwali ${ }^{20}$ showed an "urgent need" for educational programs to promote correct oral hygiene. Furthermore, a study found that the Pakistani population-specifically those of lower socioeconomic background hold belief in various myths regarding oral hygiene practices and often rely on home remedies rather than visiting adentist ${ }^{21}$. As mentioned earlier, similar studies done in western countries showed better results, and here the fault may lie in the dental curriculum. Oral hygiene and preventive dentistry are studied under the subject "Community and Preventive Dentistry" in second year of dental school, in accordance to PM \& DC curriculum ${ }^{22}$. However, 
many western universities provide multiple courses focused on patient care, preventive dentistry, and oral hygiene ${ }^{23}$. Often, these subjects are taught in preclinical (as early as first year) and clinical years ${ }^{24}$. Hence, there may be a need to revise the curriculum, but further research by subject specialist is needed.

The small sample size is a limitation of this study; therefore, a multi-centered study would show more conclusive results. Also, a longitudinal study where the result of first year students was followed up in final year would have been the gold standard. However, as there is a uniform admission criteria and students belong to more or less similar socioeconomic backgrounds this may not be a serious confounding factor.

\section{RECOMMENDATIONS}

It is recommended that there should be greater emphasis at PM\&DC level on the importance of oral hygiene practices for undergraduates, so that students not only improve their own practices, but also promote correct oral hygiene practices to their patients early in their career.

\section{Disclosure}

This study was presented at 3rd Research Gala, Riphah Islamic International Medical College 2019.

\section{CONCLUSION}

Final year dental students showed significant improvement in 2 out of 7 recommended oral hygiene practices. This shows that overall, there is no statistically significant improvement in the oral hygiene practices of dental students in FUCD as they progress through university despite gaining more knowledge and practical experience over the years.

\section{CONFLICT OF INTEREST}

This study has no conflict of interest to be declared by any author.

\section{REFERENCES}

1. ADA Policy - Definition of Oral Health. Available from: https:// www.ada.org/en/about-the-ada/ada-positionspolicies-and-statements/ada-policy-definition-of-oral-health
2. Naseem S, Fatima SHH, Ghazanfar H. Oral hygiene practices and teeth cleaning techniques among medical students. Cureus 2017; 9(7): 1487.

3. Harchandani N. Oral health challenges in Pakistan and approaches to these problems. Pakistan Oral Dent J 2012; 32(3): 497-501.

4. Halboub ES, Al-Maweri SA, Al-Jamaei AA, Al-wesabi MA, Shamala A, Al-kamel A, et al. Self-Reported oral health attitudes and behavior of dental and medical students, Yemen. Glob J Health Sci 2016; 8(10): 143.

5. Hashim NT, Al-Shiekh L, Muhammed ME, Muhammed AE, ElHuda MA. Evaluation of dental students' oral hygiene attitude and behavior using HU-DBI in Sudan. Sci Postprint 2015; 1(2): e00040.

6. Gupta S, Saxena S, Sikka N, Bhatia G. Oral health attitude, knowledge, and behaviour of dental students of Jaipur, Rajasthan: A comparative study. J Indian Assoc Public Heal Dent 2015; 13(4): 459-63.

7. Peker K, Uysal Ö, Bermek G. Dental training and changes in oral health attitudes and behaviors in istanbul dental students. J Dent Educ 2010; 74(9): 1017-23.

8. Jimbo R, Scaglia P, Niknamdeh A. Assessment of current oral health knowledge attitude and oral hygiene practices among 12-year old school children and patients attending the dental facility at Vezo Hospital in the rural village of Andavadoaka, Madagascar. Bachelor Thesis Malmo University Madagascar 2017. Available from: https://www.semanticscholar.org/paper / Assessment-of-current-oral-health-knowledge-and-old-ScagliaNiknamdeh/d5c320e660a108a0cea00a93fd01715e2f546d85

9. Inaam A, Mansoor QN, Maham N, Tayyaba S, Khalid T. A survey of oral hygiene practices amongst dental students. Pakistan Orthodontic J 2017; 9(1): 50-55.

10. Rong WS, Wang WJ, Yip KHK. Attitudes of dental and medical students in their first and final years of undergraduate study to oral health behaviour. Eur J Dent Educ 2006; 10(3): 178-84.

11. Halboub ES, Al-Maweri SA, Al-Jamaei AA, Al-wesabi MA, Shamala A, Al-kamel A, et al. Self-reported oral health attitudes and behavior of dental and medical students, Yemen. Glob J Health Sci 2016; 8(10): 143.

12. Vangipuram S, Pallavi S, Radha G, Rekha R. Assessment of oral health attitudes and behavior among undergraduate dental students using Hiroshima University Dental Behavioral Inventory HU-DBI. J Indian Assoc Public Heal Dent 2015; 13(1): 52-55.

13. Barrieshi-Nusair K, Alomari Q, Said K. Dental health attitudes and behaviour among dental students in Jordan. Community Dent Health 2006; 23(3): 147-51.

14. Rimondini L, Zolfanelli B, Bernardi F, Bez C. Self-preventive oral behavior in an Italian university student population. J Clin Periodontol 2001; 28(3): 207-11.

15. Frequency of dental $\mathrm{fl}$ ossing in studying samples. Indian J Dent Res Available from: http://www.ijdr.in/viewimage.asp?img= IndianJDentRes_2010_21_1_84_62820_t2.jpg

16. Cebeci NÖ, Nemli SK, Ünver S. Oral health behavior differences between dental students in graduate and doctoral programs. Eur Oral Res 2018; 52(1): 5-11.

17. Polychronopoulou A. Oral self-care behavior among dental school students in Greece. J Oral Sci 2002; 44(2): 73-78.

18. Khami MR, Virtanen JI, Jafarian M, Murtomaa H. Oral health behaviour and its determinants amongst Iranian dental students. Eur J Dent Educ 2007; 11(1): 42-47.

19. Barrieshi-Nusair K, Alomari Q, Said K. Dental health attitudes and behaviour among dental students in Jordan. Community Dental Health 2006; 23: 147-51. 
20. Gulzaib, Hussain P, Anjum QA, Iqbal Z. Oral hygiene awareness among the patients visiting the dental centers of a rural area of the province Punjab, Pakistan. Biomedica 2017; 33(1): 20-24.

21. Khan SA, Dewani N, Bilal S. Perceptiona and myths regarding oral health care amongst strata of low socioeconomic community in Karachi, Pakistan. J Pakistan Med Assoc 2012; 62(11): 1198-203.

22. Curriculum of BDS prepared by Pakistan Medical and Dental
Council \& Higher Education Commision Islamabad [Internet]. [cited 2019 Dec 29].

23. Curriculum Overview. College of Dentistry. University of Florida. Dental.ufl.edu. 2020. Available from: https://dental.ufl. edu/education/dmd-program/curriculum-overview/

24. Kings College London. Dentistry BDS (Bachelor of Dental Surgery) 2018. Available from: kcl.ac.uk/study/undergraduate/ courses/dentistry-bds.aspx\%0AOur 